\title{
REVIEW
}

\section{THE CLINICAL ROLES OF PSYCHOLOGISTS IN END-OF-LIFE CARE: A NEW HORIZON IN INDIAN PALLIATIVE CARE}

\author{
Vaiphei Suantak Demkhosei, Sisodia Devendra Singh
}

\author{
Bhupal Nobles' University, Department of Psychology, Udaipur, India
}

\begin{abstract}
Among all the various existing skills needed in palliative end-of-life care, none is more important the ability to communicate effectively. Terminal ill experience is the time when patients were overwhelmed with several emotional and psychological symptoms, which need the maximum amount of care, through effective communication in the most appropriate way. Effective communication is the fundamental component to physician and patient quality relationship, which make clinician to be trusted by the patient, resulting in quality decision making for patient treatment and future diagnosis plan in the clinical practices. However, looking at the present scenario of Indian health care system, the absence of good communication is visible in most of the clinical practices, which becomes the underlying reason for terminal patient receiving unwanted life sustaining medical treatment, along with inappropriate care that goes against the patient's choices. Moreover, effective communication also plays an important role in breaking the bad news to patient and family. Thus, seeing at the urgent needs of effective communication in the clinical practices, the present study is forms through fieldwork that is follow up by an analytical study. The aim of this particular research is to inquire, how important the effective communication is in terminal care, and what are the strategies for breaking bad news effectively without hurting the feelings of the dying individual's.
\end{abstract}

\section{Introduction}

There is no easy way to deliver quality of life for those experiencing impossibility of cure in their terminal illness experiences. Especially, in a country like India which is the cantered of world cancer mortality and the highest contributor's to the world cancer death at present scenario as per the World Health Organization report. However, the palliative end-of-life care primary aim is to let terminal ill patients experiencing meaning in illness by any possible means in the clinical practices. To enables the patient to have quality of life through quality end-of-life care, and to deliver patient preference treatment. Caring the distressing pain symptoms, feeling of discomfort, emotional suffering, and mental disharmony in clinical setting require maximum amount of cares, efforts, attentions, which are hardly visible in the India palliative end-of-life care at present. Majority of the Indian clinicians working in the palliative end-of-life care failed to possess these requirements in their clinical practices, resulting in leaving the terminal ill patients drowning in their emotional sufferings and mental disharmony. The miserable conditions of the terminal ill patients in the clinical practices of the country can be minimize effectively only through when the psychological, social, and spiritual approach to terminal care had been deliver alongside the contemporary medicinal treatments. The intervention of psychological approaches is essential to deliver the whole person treatment for the well-being of the whole rather than focusing in the physical pain and symptoms alone. The effective implementations of the psychological approach to terminal care can restore patients hope, quality of life, and patient's anxiety over inevitable death, which usually occurs in the terminal experiences of the terminally ill patients. In a country like India, where more than two lakhs people died per day without experiencing quality of life and well-being of the whole the psychotherapeutic approach to care will successfully encounter several psychological symptoms of a dying patient. Moreover, the psychotherapeutic techniques of pain management and relaxation therapy could be a great helps not only to the patients, but also to the family and the clinicians as a whole that will gives a new horizon towards the palliative end-of-life care of the country. 
Moreover, the underlying objectives of palliative care does not confine in bodily pain and symptoms treatment alone, rather it sought for well being of the whole with quality of life. The whole person treatment includes physical pain, psychological sufferings, social stigma, and fulfilling spiritual needs of the terminal patient in the clinical practices. Thus, considering the urgent needs for implementations of the psychotherapeutic approach to care in Indian palliative endof-life care, the current study is form through fieldwork with an analytical study. The study also consist a critical review on the available documents.

\section{Why Psychologists in Palliative End-of-Life Care?}

Living with dignity is the constitutional right given to every Indians by the law of the country. However, in most of the existing palliative end-of-life care centres in the country, the mental disharmony, stress, depression, emotional sufferings were left unconcerned that gives the terminal patients lives with no values. The prime focus in Indian palliative end-oflife care is on physical pain and symptoms, in which the psychological symptoms in terminal experience are not consider as part of terminal illness that leaves the patient in hopeless and helpless conditions. However, as per the concepts of WHO the palliative end-of-life care is a specialized area of healthcare given to any terminal ill patients, in order to prevent unwanted experiences and for relieving the patients from mental and physical pain related sufferings by any means. However, the core aim that is to produce quality of life through the whole person treatment is sometimes not visible in the clinical practices of the country, mainly due to the absence of multidisciplinary team. The palliative end-of-life care is a multidisciplinary critical care approach, which serve as a healing therapy towards terminal ill patients, which in India is provides mainly by physicians alone in the absence of any other care providers like psychologists and social workers, confining mainly to contemporary medicinal realm alone. However, Tanchel stated that in clinical practice meeting the psychological needs of all those involves and understanding the psychological dimension to the work in palliative end-oflife care strengthen the contributions of any professionals with quality care outcomes (1).

To Dawning, palliative end-of-life care is a specialized medical care provided at any given point in the trajectory of an illness with an aim to alleviate terminal ill patient physical, psychological, and social sufferings to enhance quality of life in any possible means. The purpose is to manage symptoms effectively through comprehensive interdisciplinary supports given to the terminal patient and family throughout the courses of illness, regardless of cancers of all stages in the clinical practices (2). However, looking at Indian palliative end-of-life care it is visible as a mere medical treatment given to the terminal ill patients in separate ward that requires the activity of physicians alone, in which the involvement of professional psychologists are neglects to the most. This makes the palliative end-of-life care in the country failing to produce quality of life leaving emotional sufferings and mental disharmony untreated, which were the major symptoms in terminal illness, rather than the physical pain symptoms. However, the inclusion of processional psychologists in clinical practices can give the certainty of healing the whole person even when cure is not possible and will helps the physicians to achieve the best possible quality of life for the patient and family in the best possible ways with quality end-of-life care in the country. The clinician role of psychologists can prevent the terminal ill patients from those treatments that violate their preferences, preserving patient's autonomy, values, and dignity, which will enhances the terminal patient conditions and well-being by any means through its psychotherapeutic techniques (2). Implying the said techniques in the terminal illness treatments policy, it will create the bringing of a new horizon of care in the country.

\section{The Significances Roles of Psychologists in Clinical Practices}

In a country like UK, Canada, United States, Australia, and Iceland, professionals psychologists plays a significant role in palliative end-of-life care alongside the clinicians in the clinical practices, resulting in producing quality of life for those with terminal illness. At present, in the developed countries, the psychologists are increasingly in demand in the health care systems, especially when cure is not possible in the course of an illness. However, the involvement of the psychologists in palliative end-of-life care in India is visible minimal, though the rate of terminal illness in the country grows rapidly and at present becoming the world top contributor's to cancer mortality. The WHO highly emphasis on the effective interventions of psychotherapeutic approaches to palliative care alongside the treatment of physical pain and symptoms, it urges to ensure the psychological well being of the patient and family by health care providers (3). To Vaiphei et al, the inclusion of psychologists in palliative care team is essential for quality psychological assessment to address the complex emotional and mental trauma, which is oftenly experience by terminal ill patients (4). In palliative end-of-life care, the psychotherapeutic approach to terminal care could be effective mostly before the terminal patient critical condition strike, during the terminal diagnosis, even in the advance stage of terminal illness and for the bereavement policy as noted by several studies.

We are in the era where death is no longer confines in home atmosphere anymore, it has been institutionalize with several therapeutic techniques in hospitals, old age home, and other clinical care centres aiming to delivers quality end-of-life care with good and meaningful death. Thus, assisting health care providers by practitioner or clinical psychologists becomes essential to uplift the standard of palliative end-of-life care in the country and to meet the patient needs and requirements. The Psychologists in clinical practices can plays the essential roles of an advocate, counsellor, educator, evaluator, and the researcher that would enhances the quality of care and treatments. The role of psychologists are not limited to the patients alone, it could effectively manage the terminal patient family well being conditions and the stressful events of the clinicians through supportive counselling psychotherapy $(5,6)$. The outcomes of the psychologists in terminal treatments will 
gives the physicians quality treatment decision, which is in line with the patient choices, helps in adjusting difficult situations, makes patient emotionally strengthen to face death anxiety and to create an awareness for general preparations for death and bereavement policy.

The psychotherapeutic approach to the terminal illness and terminal care is a patient preference structure of care in the face of medical helplessness (7). It is only through the psychotherapeutic therapy that acknowledges the patient nature of distress. It examines the hopelessness, and the burdensome experiences causes through terminal illness (8). Moreover, when cure is not possible in clinical practices the psychotherapeutic approach can restore patient sense of meaning, hope, value, and dignity that usually gives a peaceful death to patient and helps the patient family in their bereavement period. Moreover, many existing researches highlights the psychotherapeutic approach to terminal care is an effective coping mechanism against terminal ill experience of any stage, which helps the patient's and the clinicians to avoids having wrong perception to cure in the clinical practices. The time when clinicians mostly needed the psychologists, in palliative endof-life care is mainly when it comes to the patient mental disharmony and a hopelessness experience $(9,10)$. Moreover, the care for the terminal ill patients demands an extensive amount of care, which the doctors and nurses alone cannot manage effectively. The terminal ill treatment is not confine in the physical pain and symptoms alone; it rather requires several mental and emotional treatments, which are beyond the riches of the contemporary medicines and its technologies. In terminal diagnosis, the psychologists have the professional capability to reduce the tense situation through its good communicator and comforter role. The psychologists can also helps in building a quality relationship between a patient and the care providers and with the patient's family to come together for meaningful discussion in any possible ways in regards to patient treatment, plan, and the bereavement policy.

The intervention of clinical or health psychologists in a terminal treatment can helps the patient in controlling the physical symptoms and in providing supportive presence to the patient's to overcome isolation. It can help the patient to recognize his/her value, meaning, purpose, reconciliation with self, others, and loved ones. The psychotherapeutic involvement in terminal treatment will help the patient to reframe his/her life goal through self-reflective life review psychotherapy (11). Thus, the psychologists can rebuilt and reconstruct the present patient and clinicians' communication barriers to uplift the Indian palliative endof-life care to the new horizons. Moreover, the psychologists are train to be an effective encourager's, so the involvement of the psychologists in the clinical practices of the country could successfully play the role of the patient's guides to perform a meditative practices, which will helps the patient to focus on healing of the whole even when cure is not possible in his/her diagnosis. The psychotherapeutic intervention can provides the certainty of terminal patient being respect and preserve personal valued, which help patient to view life with positive, which is still in the condition of limitless achievement apart from being ill. Such therapeutic intervention is what Indian palliative end-of-life care needs, which could give meaning in sufferings and will be able to gives the two lakhs terminal patients dying per day in the country. However, the expert interventions in dealing with disappointment, death anxiety, sense of hopelessness, a patient-cantered therapy for meaning making process are not visible in Indian palliative end-of-life care at present. Thus, the unavailability's emotional sufferings and mental disharmony in Indian terminal treatments resulted in worsening the conditions of the terminal ill patient in most cases.

The Indian health care systems as a whole, and palliative end-of-life care at particular, needs to learn from the British Psychological Society concerning the terminal ill treatments and its bereavement policy for effective end-of-life care in the country. In terminal ill treatment, the British Psychologists acknowledges that the terminal experience is preoccupied with several unwanted negative feelings, loss of sense and dignity. Not only the patient's, even the clinicians also experience several psychological difficulties in caring for the terminal ill patients that becomes major risk to palliative end-of-life care, which makes the Psychological problems as the essential component in clinical practices (12). Thus, the Indian palliative end-of-life care can only be fruitful and effective only when psychologists treats the existing psychological problems like depression, mental disharmony, and the several anxieties that are associated with the pending death. The grief psychotherapy and counselling can provides compassionate care towards the patient and family. Moreover, the psychologists can helps the patient and family to understand the confusing medical terms, terminal diagnosis procedures, the effects of Chemotherapy and dealing with the clinician's stressful moment effectively in the clinical practices. The researcher also would like to implement Backer's understanding of the significant roles of psychologists in delivering quality end-oflife care towards the clinical practices of the country. The psychologists are not only effectives with patient's depression, emotional and mental disharmony, but they can facilities several psychological problems of the 'trio' (patient, family, and clinician) in the clinical practices through their biopsychosocial-spiritual psychotherapeutic techniques (13). Moreover, the psychologists in health care systems of the country can play an effective role of a researcher to explore on several major issues around terminal illness and palliative end-of-life care, which could helps in advance quality health care planning, quality decision-making, and exploring the latest aspects of psychological pain and symptoms management. The involvements of psychologists in the clinical practices of the country can ensure the reliability of palliative end-of-life care in the battlefield against the cool blooded killer 'Terminal Illness.' The involvement of professional psychologists in Indian palliative end-of-life care, the terminal diagnosis could see its new horizons within a decade. The process of undergoing the terminal diagnosis 
and chemotherapy is the time when the patient's are overwhelms with several sentimental crises of living or dying. It is the time when they need the psychological intervention the most and psychotherapeutic approach to care at this point is visible the most effective mechanism in terminal experience, which the Indian palliative end-of-life care failed to delivers to the terminal patients in the country thus far. This being the reason, India is not a good place to die, where patient's preference care were not meet, patient's dignity unconcerned, and the voice of the drying's were not been listened. The psychological approach to terminal care and treatment could delivers patient preference's of care, treatment, helps patient to make sense of their terminal diagnosis, can provides social and spiritual services effectively $(14,15)$. The psychological intervention could prevent terminal patients from symptoms worsening his/her condition and could help's in preventing the terminal patient from disability of the organ system, which usually occurs in the last hour's terminal experience's.

\section{Findings and Practical Challenges}

The psychologists had great roles to play in Indian palliative end-of-life care though dealing with diseases and its therapeutic process is not an easy task. The one reason why psychologists are being neglect in the health care systems of the country is the concept of the people, "psychologists are not medical practitioner who does not have well to do knowledge with the contemporary medicines and its trajectories." Such concepts prevail, as majority of the Indians could not differentiate the role of psychologists and psychiatrists in the clinical practices and lack of knowledge about the role and outcomes of psychotherapeutic interventions in terminal illness. At present, it will take a decade or two for Indian to realize the importance of psychologists and its psychotherapeutic techniques in the clinical practices of the country. The modern medicine and equipments cannot cure symptoms like depression, loneliness, emotional sufferings, stress, mental disharmony, feeling uneasiness, and death anxiety. It needs psychological interventions to treat those psychological symptoms, which usually arose in terminal patient's experience. On the hand, the Indian psychologists also need to create an awareness platform where they can earn people trusted the same way they trust and rely on other health care providers in the country.

Moreover, for effective functioning, the psychologists working in clinical realm need to have the basic knowledge on the cause's cancers. What terminal illness is all about? What are the types of symptoms? How terminal illness diagnosed in the clinical setting? Quill suggested that, every psychologist involve in palliative end-of-life care, need to undergo a basic study of pathophysiology, which is the physiology of abnormal states, especially the functional changes that accompany a particular syndrome or disease (16). It is essential for the psychologists to be aware of the disease processes of the patients they are assisting, mainly on some of the ongoing illness process like cancer, organ failure, progressive neurological condition and frailty, organ functioning, patient autonomy, and mental clarity, which. will enhance them in effective therapeutic approach to terminal ill patient. The psychologists should pay attention towards the physical and mental well being condition of the patient, family, and the clinicians. The psychologists assisting a terminal patient is requires to have a knowledge on the use of opoiod, the constitutional laws with regards to terminal treatment policies, quality medical treatment types, and should be fully aware of the bereavement policy. The clinical assessment of a psychologist does not end with the death of the patient; it rather is an ongoing process until the bereaved family psychologically and physically conditions stabilized. In this therapeutic process, the psychologists need to be an effective communicator, a good listener, and should possess the ability to manage their own emotions with the ability to built quality relationship at any given circumstances. The psychologists are also advice to have a basic knowledge on medical symptom management and its therapeutic techniques, along with lost and grief therapeutic techniques experiences. Kasl-Godley advice the psychologists working in palliative care to be aware of the patient unspoken emotional challenges, which were usually, inherit in the course of terminal ill experiences. There is a danger in not treating the patient unspoken emotional challenges, leading to suicide, and ineffective of the terminal medication and diagnosis (10).

It is essential for the psychologists to have sufficient knowledge on biopsychosocial-spiritual psychotherapeutic techniques and methods to delivered effective psychotherapeutic in the clinical practices, along with the ability to identify patient emotional or mood conditions, desire, and the ability to solve conflict issues or situation in any given context. The psychologists are also requires to possess the discerning capability to identify family and clinician present condition and the capability to recognize the types of treatment the patient and family wants their clinician to performed for effective treatment and fruitful psychotherapy. The challenging roles of the psychologists working in the clinical setting is the time when their terminal ill patients are not in the condition to makes his/her decision and the family members are in confusion state concerning which decision to make for their loved ones. Especially, when it comes to euthanasia, the used of opoiod, and other critical conditions, the psychologists are mostly required to assist the patient and family for quality decision-making. In which, any wrong decision advice to either patient or family can lead the psychologist into lifetime problem in his/her career. Therefore, it is essential for the psychologists to be wise in their assessment and to be aware of the assessment outcomes before assisting. The psychologist working in palliative end-of-life care need to be aware of the psychotherapeutic assessment he/she gives to patient and family, because it is not just a therapy rather a critical care medicine, which has its own side effect like a wrong medical prescriptions.

However, looking at the Indian health care system at present, clinicians mostly neglected the psychological wellbeing of the patient and family. The attention of the physician mostly confined within the bodily treatment, which has no concern for the emotional state of the 
However, looking at the Indian health care system at present, clinicians mostly neglected the psychological wellbeing of the patient and family. The attention of the physician mostly confined within the bodily treatment, which has no concern for the emotional state of the terminal patient as a whole, it is also visible that health care providers do not give importance for quality talk or meaningful conversation with the patient and family. In doing that, the terminal patient and family could find great relief from their burdensome experience, leading to effective treatment and care in palliative end-of-life care of the country. Moreover, as per the researcher experience, most of the hospital policy is to restrict doctors and nurses towards frequent conversation, and not to be too close with the patient and family in clinical practices. This could be the reason of Indian health care providers failing to provide or understand patient mental well being, to produce quality treatment and quality end-of-life care. Another reason is the concept of the Indian as a whole towards psychotherapy, the Indian understanding of psychotherapeutic is mostly with the abnormal behaviour or patients, in which the normal patient does not approach psychologists, and need not require psychological treatment, which is totally a wrong concept that existed in the thinking of most Indian. However, changing this concept could bring quality of life with healthy living.

The understanding of pain management confining only in contemporary medical realms should also be corrected, the psychologists and its psychotherapeutic approach to health care has huge contribution in pain management, it as a critical medicine deliver healing in the face of contemporary medical helplessness, deliver mental well being that produce healthy lifestyle. The psychotherapeutic intervention can also gives people medical and health awareness, especially in the case of terminal or incurable illness experience, providing self-compassion, letting patient being aware of the present condition, and what can comes in future. Overall, as Vaiphei et al noted, the intervention of psychotherapy will helps the terminal patient to understand the deeper meaning of life through self-reflective review on one's life, and prepare the patient to face the inevitable death with confident, and deliver peaceful death (4).

Conflict of interest: We declare that there is no conflict of interest.

Funding source: There is no funding in this article.

\section{References}

1. Tanchel Irene. Psychosocial Issues in Palliative Care. CMEContinuing Medical Education 2003;21(5):249-52.

2. Downing J. Editorial: To Research or Not To Research - An Important Question in Paediatric Palliative Care. Palliative Medicine 2016;30(10):902-3.

3. World Health Organization. Palliative Care. http://http://www.who.int/news-room/fact-sheets/ detail/ palliative-care. Access Date: 30th July 2018.

4. Suantak Demkhosei V, Singh Devendra S. The emerging needs for biopsychosocial-spiritual model in Indian palliative end-of-life care: An analytical study. Journal of Well Being 2018;12(2):20-5.

5. Werth JL, Gordon R, Johnson RR. Psychological issues near the end of life. Aging Ment Health 2002;6(4):402-12.

6 . Kaut KP. Religion, spirituality, and existentialism near the end of life: Implication for assessment and application. Journal of American Behaviour Scientist 2002;46(2):220-34.

7. Chochinov HM. Dying, dignity, and new horizons in palliative end-of-life care. CA Cancer J Clin 2006;56(2):84103.

8. Puchalski CM. Spirituality and end-of-life care: A time for listening and caring. J Palliat Care Med 2002;5(2):289-94.

9. Meador KG. Spiritual care at the end of life: What is it and who does it? NC Med J 2004;65(4):226-8.

10. Kasl-Godley JE, King DA, Quill TE. Opportunities for psychologists in palliative care: Working with patients and families across the disease continuum. Am Psychol 2014;69(4):364-76.

11. Rousseau P. Spirituality and dying patient. J Clin Oncol 2000;18(9):2000-2.

12. The British Psychological Association. Division of Clinical Psychology Faculty of Clinical Health Psychology: Briefing Paper No. 27 Clinical Health Psychologists in the NHS. http://www1.bps.org.uk/system/files/Public\%20files/DCP/c at-442.pdf. Access Date: 08th June 2017.

13. Backer A. Palliative and end-of-life care in the serious and persistently mentally ill population. J Am Psychiatr Nurses Assoc 2005;11(5):289-303.

14. William HE, Larson DG, Kasl-Godley J, Neimeyer RA, Kwilosz DM. The roles of psychologists in end-of-life care: Emerging models of practice. Journal of Professional Psychology: Research and Practice 2003;34(6):626-33.

15. DeAngelis T. More psychologists needed in end of life care. Journal of American Psychologists 2002;33(2):49-52.

16. Quill TE. Hospice and Palliative Medicine Visionary Timothy Quill Shares His Insights on the Field. American Academy of Hospice and Palliative Medicine. http://aahpmblog.org / articles / hospice-and-palliativemedicine - visionary - timothy - quill - shares - his insights -on-the field/. Access Date: 14 ${ }^{\text {th }}$ July 2018. 